\title{
Role of Nuclear Factor Erythroid 2-Like 2 in the Induction of Cytochrome P450 2a5 in Vivo of Nonalcoholic Fatty Liver Disease
}

\author{
Yizhe Cui ${ }^{1,2}$, Qiuju Wang ${ }^{2}$, Chunhua Wang ${ }^{1}$, Xing Yi ${ }^{1}$, Yue $\mathbf{Q i}^{1}$, Guangliang $\mathrm{Li}^{1}$ and Xiuying Zhang ${ }^{\text {* }}$
}

${ }^{1}$ College of Veterinary Medicine, Northeast Agricultural University, Mucai Street, Xiangfang District, Harbin 150030, Heilongjiang, China

${ }^{2}$ College of Animal Sciences and Technology, Heilongjiang Bayi Agricultural University, Xinyang Road, New Development District, Daqing 163319, Heilongjiang, China

\begin{abstract}
Mouse cytochrome P450 2A5 (Cyp2a5) is upregulated in various pathophysiological liver disease and induced by structurally variable hepatotoxic chemicals. A putative common feature for all of these conditions is altered cellular redox status. Nuclear factor erythroid 2-like 2 (Nrf2) is a transcription factor that is post-translationally regulated by oxidative stress and controls the transcription of numerous protective target genes. In order to determine if upregulation of Cyp2a5 by Nonalcoholic fatty liver disease (NAFLD) was mediated through Nrf2, we applied Nrf2 knockout mice ( $\mathrm{Nrf}^{-}$) to examine the Cyp2a5 mRNA and protein levels compared to wild type mice. Furthermore, the interaction of Nrf2 with Cyp2a5 was confirmed by co-immunoprecipitation (co-IP) in vivo. The NAFLD increased the Nrf2 mRNA and protein levels in wild type mice and also increased the Cyp2a5 mRNA and protein levels resulted from translocation of Nrf2 into the nucleus. However, the expression and activity of Cyp2a5 were no difference in the Nrf2 knockout mice. Nuclear immunohistochemical staining of Nrf2, an indicator of activation of the transcription factor, was evident in NAFLD. Co-IP experiments showed that Nrf2 binds to the Cyp2a5. Our current results unequivocally show that expression of Cyp2a5 is tightly controlled by Nrf2 in liver. Nrf2 is needed for constitutive expression of Cyp2a5, and Cyp2a5 is also sensitively upregulated by an increased level of Nrf2 protein. Therefore, Cyp2a5 upregulation could be a useful indicator for hepatic activation of the Nrf2 pathway.
\end{abstract}

Keywords: Coumarin 7-hydroxylase; Oxidative stress; High-fat diet; Knockout mice; Pyrazole

Abbreviations: ARE: Antioxidant Response Element; Nrf2: Nuclear Factor Erythroid 2-like 2; KO: Knockout; WT: Wild-Type; P450: Cytochrome P450; NASH: Non Alcoholic Steato Hepatitis; NAFLD: Non Alcoholic Fatty Liver Disease; co-IP: Co-Immuno Precipitation

\section{Introduction}

Nonalcoholic fatty liver disease (NAFLD) composes a spectrum of etiologies ranging from simple fatty liver (steatosis) to the more severe Nonalcoholic Steatohepatitis (NASH). The proposed mechanism for progression of NAFLD involves a two-hit theory where lipid accumulation in hepatocytes (the "first hit") is followed by a "second hit," including insulin resistance, oxidative stress, and cytokine production [1]. Several of the factors regulating hepatic CYP2E1 and CYP4A expression have been linked to the pathogenesis of hepatic steatosis and NASH. In the context of hepatic steatosis, both CYP2E1 and CYP4A generate the "second hit" of cellular injury, particularly when antioxidant reserves are depleted [2]. In contrast, relatively little data has been reported with respect to the effect of NAFLD on hepatic Cyp2a5.

The mouse Cyp2a5 and its human ortholog CYP2A6 mediate the phase I metabolism of thousands of endogenous and xenobiotic substrates, including environmental pollutants, pharmaceuticals, steroids, prostaglandins, and fatty acids [3]. It is induced by classical inducers, such as pyrazole and carbon tetrachloride [4,5]. Elevated CYP2A5/CYP2A6 protein levels in hepatocytes are associated with the development of liver tumours in mice [6] and in humans [7] respectively. It has been proposed that Cyp2a5 may be one of the major xenobiotic-metabolizing CYP forms in a damaged liver [8]. However, till now, the mechanism(s) by which these xenochemical inducers and pathophysiological stressors, such as oxidant stress, regulate hepatic Cyp2a5 in mouse tissues are poorly understood, and little data has been reported with respect to the effect of NAFLD on hepatic expression of Cyp2a5.

Recent studies have indicated that induction of Cyp2a5 is not directly related to the nature of the inducing agents, but instead may be an indirect consequence of a specific cellular event associated with the pathogenesis of liver injury. For example, certain agents may disturb cellular redox status, a common denominator that may consequently induce the expression of CYP2A5 through activation of stress-related transcription factors, such as the transcription factor nuclear factor E2-related factor 2 (Nrf2) [9-11]. Nrf2 is primarily a cytosolic protein due to proteasomal degradation regulated by Keap1 $[12,13]$. When a cell is undergoing oxidative stress, Nrf2 escapes Keap1-mediated degradation, translocates to the nucleus, binds to ARE sequences within the promoters of target genes, and induces expression of phase I and II metabolizing enzymes and transporters pivotal to the maintenance of oxidative stress-inducing molecules [14]. Until present, it is unclear to whether induction of Nrf2 can induce Cyp2a5 and the Nrf2 interaction with Cyp2a5 in NAFLD.

The aim of the present study was to elucidate the potential role of Nrf2 in NAFLD-mediated Cyp2a5 regulation. The results show existence of Nrf2 binding Cyp2a5 and that NAFLD activates binding

*Corresponding author: Xiuying Zhang, Department of Basic Veterinary Science, College of Veterinary Medicine, Northeast Agriculture University, Muca Street, Xiangfang District, Harbin150030, Heilongjiang, China, Tel/Fax: +86 451 55190674; E-mail: zhangxiuying@neau.edu.cn

Received April 23, 2014; Accepted January 02, 2015; Published January 04 2015

Citation: Cui Y, Wang Q, Wang C, Yi X, Qi Y, et al. (2015) Role of Nuclear Factor Erythroid 2-Like 2 in the Induction of Cytochrome P450 2a5 in Vivo of Nonalcoholic Fatty Liver Disease. J Diabetes Metab 6: 488. doi:10.4172/2155-6156.1000488

Copyright: (c) 2015 Cui Y, et al. This is an open-access article distributed under the terms of the Creative Commons Attribution License, which permits unrestricted use, distribution, and reproduction in any medium, provided the original author and source are credited. 
of Nrf2 to Cyp2a5, which in turn up-regulates the Cyp2a5. The present evidence represent a novel mechanism in the regulation of phase I xenobiotic-metabolizing gene.

\section{Materials and Methods}

\section{Animal studies}

All animals received proper care in agreement with animal protocols approved by the Institutional Animal Use and Care Committee of the University of Northeast Agricultural. Six to eight-week-old, male ICR wild-type $\left(\mathrm{Nrf2}^{+/+}\right)$and Nrf2 knockout mice $\left(\mathrm{Nrf2}^{-/}\right)$mice were purchased from the Animal Experiment Center of General Hospital of Nanjing Military Area Command [Certification No. SCXK (JUN) 2007-012]. The mice were treated for eight consecutive weeks, the control group (Control) was fed with the standard diet, high-fat group (NAFLD) was fed with a high-fat feed $(68.5 \%$ standard diet, $15 \%$ lard, $1 \%$ cholesterol, $0.5 \%$ bile and $15 \%$ dextrin) [15], and the pyrazole group was used as positive control (PRY+) and fed with the standard diet but injected intraperitoneally with pyrazole $(150 \mathrm{mg} / \mathrm{kg}$ body wt) once per day in the last three days before sacrificed [16]. Twenty-four $h$ after the last pyrazole injection, the mice in all groups were euthanized with $\mathrm{CO}_{2}$ gas. The livers were removed, rinsed in ice-cold saline, and divided for various assays, as outlined below.

\section{Immunofluorescence assay}

Hepatocytes were isolated by adaptation of the calcium two step collagenase perfusion techniques as previously described $[17,18]$. Hepatocytes were plated on collagen coated six well plates at 250000 cells/well. The cells were grown on glass coverslips in 24-well plates, then were washed with PBS, fixed with $4 \%$ paraformaldehyde for $10 \mathrm{~min}$ at RT, permeabilized for 5 min with $0.2 \%$ Triton X-100 and blocked for 45 min with $5 \%$ bovine serum albumin in PBS. The cells were next incubated with primary antibodies Nrf2 (1:100) for $1 \mathrm{~h}$ at $\mathrm{RT}$, and followed by incubation with FITC conjugated goat anti-mouse IgG (1:50 dilution) for $1 \mathrm{~h}$ at room temperature. After washing with PBS, cells were mounted with an anti-fade reagent containing DAPI (4, 6-diamidino-2-phenylindole; Invitrogen) and observed under a fluorescence microscope equipped with the Nikon Metamorph digital imaging system.

\section{Immunohistochemistry}

Livers were fixed in formalin and embedded in paraffin. Deparaffinized sections $(5 \mu \mathrm{m})$ were used for immunohistochemistry. For antigen retrieval, sections were heated in $100 \mathrm{mM}$ sodium citrate, $90^{\circ} \mathrm{C}$, for $10 \mathrm{~min}$ followed by gradual cooling to room temperature. Endogenous peroxidases were inactivated with $0.3 \% \mathrm{H}_{2} \mathrm{O}_{2}$ for $10 \mathrm{~min}$ at room temperature and sections were blocked with $5 \%$ BSA in $1 \times$ PBST for one hour. Incubation with affinity purified anti-Nrf2 antibody was performed overnight at room temperature followed by standard washes and one-hour incubation with goat HRP-conjugated antirabbit IgG (Bio-Rad). Following washes, sections were developed using diaminobenzamidine at $\mathrm{pH} 7.0$ and counter-stained with hematoxylin.

\section{Isolation of nuclear and cytoplasmic proteins from mouse liver}

Nuclear and cytoplasmic extracts were prepared with Nuclear and Cytoplasmic Protein Extraction Kit according to their protocol (Beyotime Institute of Biotechnology, Beijing, China). Protein concentrations were determined with the BCA Assay Kit from Beyotime Biotechnology. Briefly, approximately $0.2 \mathrm{~g}$ of mouse liver was homogenated with $2 \mathrm{ml}$ of ice cold hypotonic buffer $\mathrm{A}$ and buffer B (20:1). The homogenate was incubated at $4^{\circ} \mathrm{C}$ for $15 \mathrm{~min}$ and centrifuged at $12,000 \mathrm{~g}$ for $10 \mathrm{~min}$ at $4^{\circ} \mathrm{C}$. The supernatant was obtained as cytoplasmic extract and the residue was added with 0.2 $\mathrm{ml}$ of hypertonic buffer $\mathrm{C}$. The pellet suspension was shaken gently for $30 \mathrm{~min}$ at $4^{\circ} \mathrm{C}$ and then centrifuged at $12,000 \mathrm{~g} 10 \mathrm{~min}$ at $4^{\circ} \mathrm{C}$. The supernatant was obtained as nuclei extract. PMSF and leupeptin were added into buffer $\mathrm{A}$ and buffer $\mathrm{C}$ before use.

\section{Immunoprecipitation}

Liver fractions were extracted as described above. Sufficient amount of Nrf2 antibody or Cyp2a5 antibody or mouse IgG negative control was added into $200 \mu \mathrm{g}$ proteins and gently rotated at $4^{\circ} \mathrm{C}$ overnight. The immunocomplex was captured by adding $25 \mu$ protein $\mathrm{A}+\mathrm{G}$ agarose beads (Beyotime, Beijing, China) and gently rotating at $4^{\circ} \mathrm{C}$ for $3 \mathrm{~h}$. Then the mixture was centrifuged at $2500 \mathrm{~g}$ for $5 \mathrm{~min}$ at $4^{\circ} \mathrm{C}$ and the supernatant was discarded. The precipitate was washed for three times with ice-cold RIPA buffer, resuspended in $3 \times$ sample buffer and boiled for $5 \mathrm{~min}$ to dissociate the immunocomplex from the beads. The supernatant was collected by centrifugation and subjected to SDSPAGE.

\section{Western blot analysis}

Approximately $0.2 \mathrm{~g}$ of mouse liver was homogenated with 1 $\mathrm{ml}$ of ice cold RIPA Lysis Buffer (Beyotime, Beijing, China). The homogenate was centrifuged at $12,000 \mathrm{~g}$ for $5 \mathrm{~min}$ at $4^{\circ} \mathrm{C}$. Supernatants were denatured; $30 \mu \mathrm{g}$ of protein was separated by polyacrylamide gel electrophoresis, and then transferred to nitrocellulose membranes (Invitrogen). Membrane blotting was performed using rabbit polyclonal antibody to $\mathrm{Nrf2}, \beta$-actin and histone $\mathrm{H} 1$ polyclonal antibody (Biosynthesis Biotechnology Co., LTD, Beijing, China) or chicken polyclonal antibody to Cyp2a5 (University of Kuopio, Kuopio, Finland).

\section{Real-time qPCR}

Total RNA was extracted using Trizol reagent ${ }^{\mathrm{m}}$ (Invitrogen, CA), followed by column purification (RNeasy ${ }^{\text {rim }}$ mini kit, Qiagen, MD) and treated with DNAse (Roche, CT). A total of $0.5 \mu \mathrm{g}$ of RNA was reverse transcribed per reaction using Sprint ${ }^{\text {tix }}$ RT Complete (Clontech Laboraties, CA). RT-qPCR was performed on ABI PRISM 7900HT Sequence Detection System (Applied Biosystems, CA). Primers sequence are as follows:

\section{Cyp2a5}

Forward 5'-GGACAAAGAGTTCCTGTCACTGCTTC-3' Reverse 5'-GTGTTCCACTTTCTTGGTTATGAAGTCC-3'

Nrf2

Forward 5'-CCA GCA CAT CCA GAC AGA CAC-3'

Reverse 5'-GAT ATC CAG GGC AAG CGA CTC-3'

\section{GADPH}

\section{Forward 5'-ACAGTCCATGCCATCACTGCC-3'}

\section{Reverse 5'-GCCTGCTTCACCACCTTCTTG-3'}

\section{Coumarin 7-hydroxylase assay}

Cyp2a5 activity was determined using the protocol described previously (18), modified for 96-well plates. In brief, coumarin 7-hydroxylase activity was assessed using $0.1 \mathrm{mg}$ of microsomal 
Citation: Cui Y, Wang Q, Wang C, Yi X, Qi Y, et al. (2015) Role of Nuclear Factor Erythroid 2-Like 2 in the Induction of Cytochrome P450 2a5 in Vivo of Nonalcoholic Fatty Liver Disease. J Diabetes Metab 6: 488. doi:10.4172/2155-6156.1000488

protein incubated with coumarin $(100 \mathrm{mM})$ and NADPH for $15 \mathrm{~min}$. The amount of hydroxylated coumarin was determined by fluorescence with an excitation wavelength of $355 \mathrm{~nm}$ and an emission wavelength of $460 \mathrm{~nm}$ using a Thermo Fluoroskan Ascent spectrophotometer.

\section{Results}

\section{Effect of high-fat diet and pyrazole on cellular distribution of Nrf2}

Cellular localization of Nrf2 protein was determined by immunofluorescence, immunostaining and Western blotting. As shown Figure 1, in untreated cells, Nrf2 fluorescence was found to be distributed throughout the cells, in both the cytoplasm and nucleus. After treatment with high-fat, most of the Nrf2 fluorescence was localized to the nuclei, as was also observed for pyrazole treatment, and immunostaining for Nrf2 showed increased nuclear staining in many hepatocytes in NAFLD as compared to control liver sections. As shown in Figure 2, a significant decrease in Nrf2 protein levels in the cytoplasm was observed after treatment with high-fat diet and pyrazole. However, Nrf2 protein levels in the nucleus were higher in high-fat diet group mice livers than that in control mice livers. These results suggested that the high-fat diet and pyrazole induced a translocation of Nrf2 from the cytoplasm to the nucleus.

Effects of high-fat diet and pyrazole on Nrf2 and Cyp2a5 expression in $\mathrm{Nrf}^{+/+}$mice

As shown in Figure 3, levels of Nrf2 were increased 24\% and $49 \%$ by high-fat diet and pyrazole compared with the mice in the control group (Figure 3A and 3B), respectively. Levels of Cyp2a5 were also were higher by high-fat diet and pyrazole (63\% and $130 \%)$. Coumarin 7-hydroxylase activity was increased approximately $71 \%$ by high-fat diet and $177 \%$ by pyrazole compared with the mice in the control group (Figure 3C). The increase in Cyp2a5 mRNA was highest treatment with pyrazole, analogous to the high protein levels and activity of Cyp2a 5 in these mice. The high-fat also elevate levels of Nrf2 mRNA in the WT mice (113\%) confirming that NAFLD induction of Nrf2 and Cyp2a5.

\section{Effects of high-fat diet and pyrazole on Cyp2a5 expression in} $\mathrm{Nrf}^{-/-}$mice

To evaluate the role of Nrf2 in high-fat diet induced Cyp2a5, levels and activities of Cyp2a5 in livers from $\mathrm{KO}$ mice were assayed (Figure 4). However, high-fat diet and pyrazole did not significantly elevate Cyp2a5 in the Nrf2 KO mice. The above studies show that NAFLD elevates Cyp2a5 protein levels and activity in WT mice but not in Nrf2 KO mice.

\section{The interaction between Nrf2 and Cyp2a5}

As shown in Figure 5, Cyp2a5 protein was detected in the protein complex from the imunoprecipitation of cell lysates using an Nrf2 antibody (Figure 5A), and Nrf2 protein also was detected in the imunoprecipitations of Cyp2a5 (Figure 5B). Furthermore, in the high-fat diet group and pyrazole group both Nrf2 and Cyp2a5 protein expression were higher significantly $(P<0.05)$ than in control group mice. There was no co-immunoprecipitation of Nrf2 with Cyp2a5 in the absence of antibody.

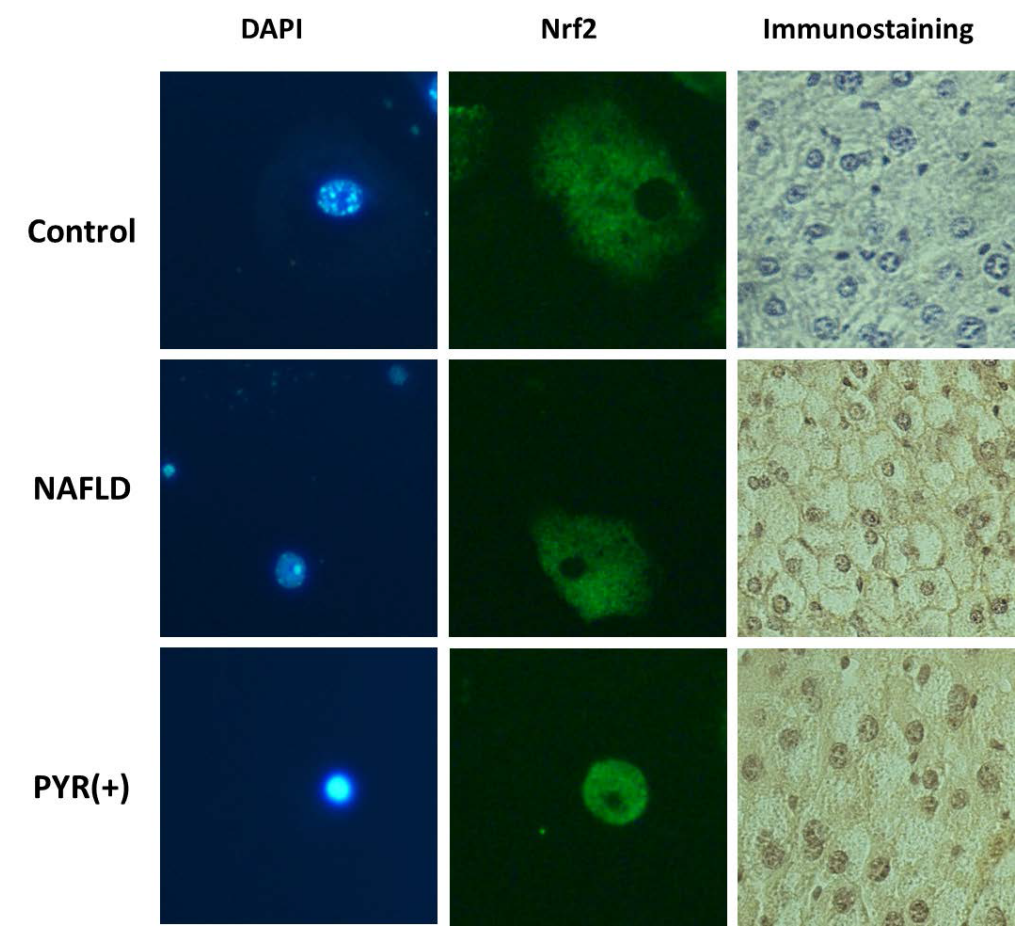

Figure 1: Nrf2 nuclear accumulation induced by NAFLD (Cellular distribution of Nrf2 obtained from the liver of mice treated with control, high-fat diet and pyrazole. An immunofluorescence assay was performed followed by detection with confocal microscopy. The cells were fixed, permeabilized and stained with antibodies against Nrf2. DAPI was used as a nuclear stain. Nrf2 specific nuclear staining by immunohistochemistry. Paraffin sections of livers of control, high-fat diet and pyrazole were stained using antibodies against Nrf2 followed by anti-rabbit horseradish peroxidase conjugate, as indicated. Chromagen development yields a brown color; sections were counter-stained with hematoxylin (blue). The increased brown staining of hepatocytic nuclei in upper panel as opposed to the more blue color of these nuclei in lower control panels indicated that Nrf2 was more abundant in high-fat diet and pyrazole nuclei. The image shows the results of a representative experiment from three independent experiments performed. Scale bars $20 \mathrm{~mm}$ ). 


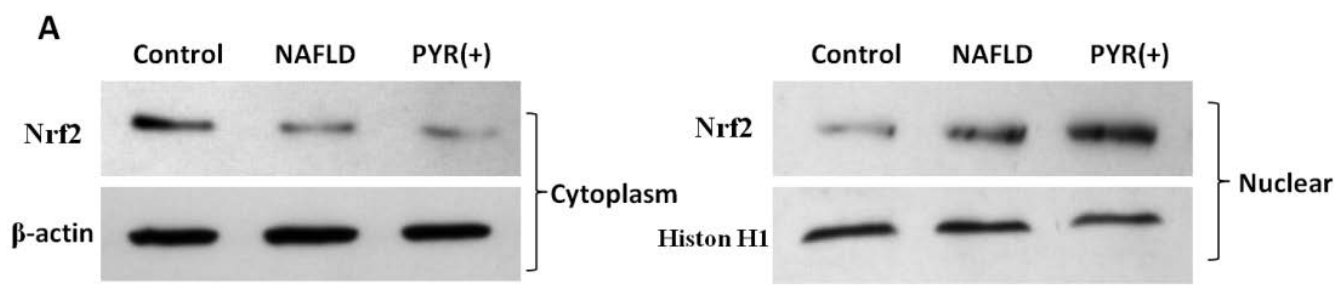

B
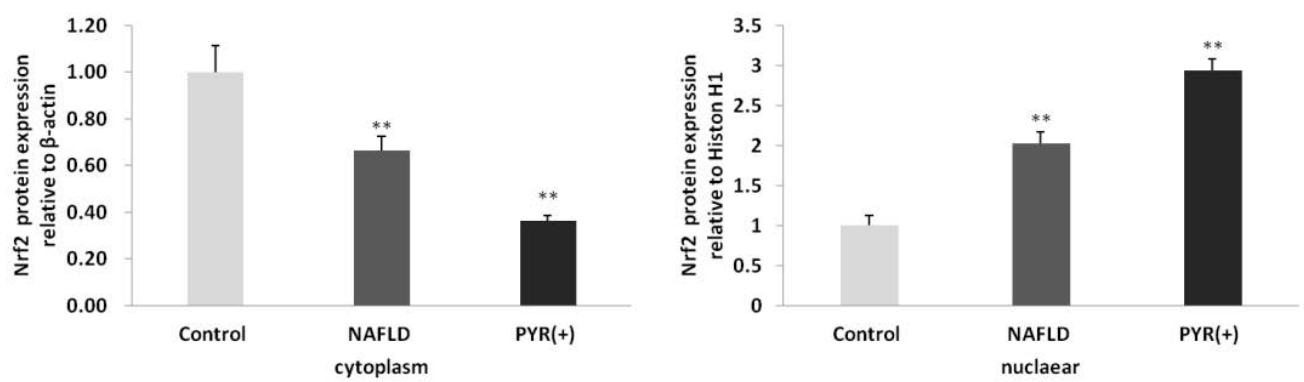

Figure 2: NAFLD induces a translocation of Nrf2 from cytoplasma to nuclear in WT mice liver (Western blot (A) and densitometric analysis (B) of Cyp2a5 and Nrf2 protein expression in the cytosolic and nuclear fractions obtained from mice treated with control, high-fat diet and pyrazole. Histon $\mathrm{H} 1 \mathrm{was}$ used as the nuclear marker. Values represent the mean $\pm S D(n=3)$. Values are significantly different from controls $\left.\left({ }^{* *} P<0.01\right)\right)$.
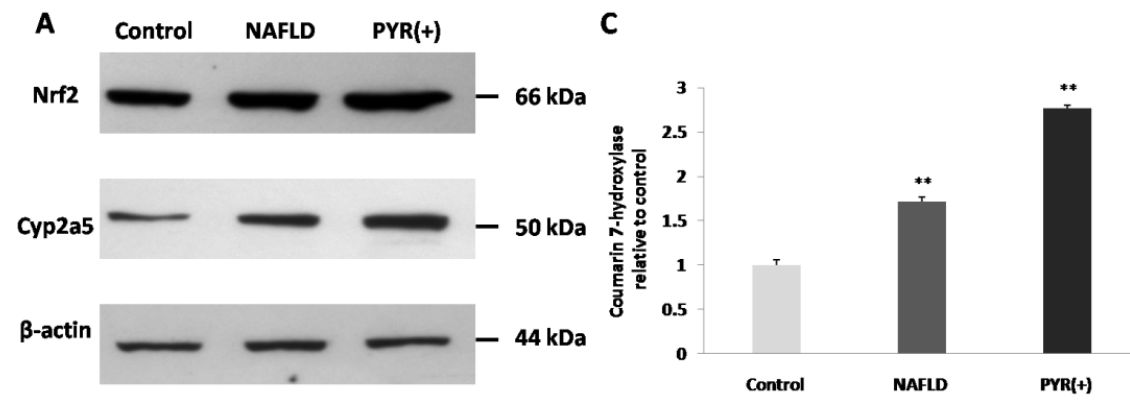

B

D
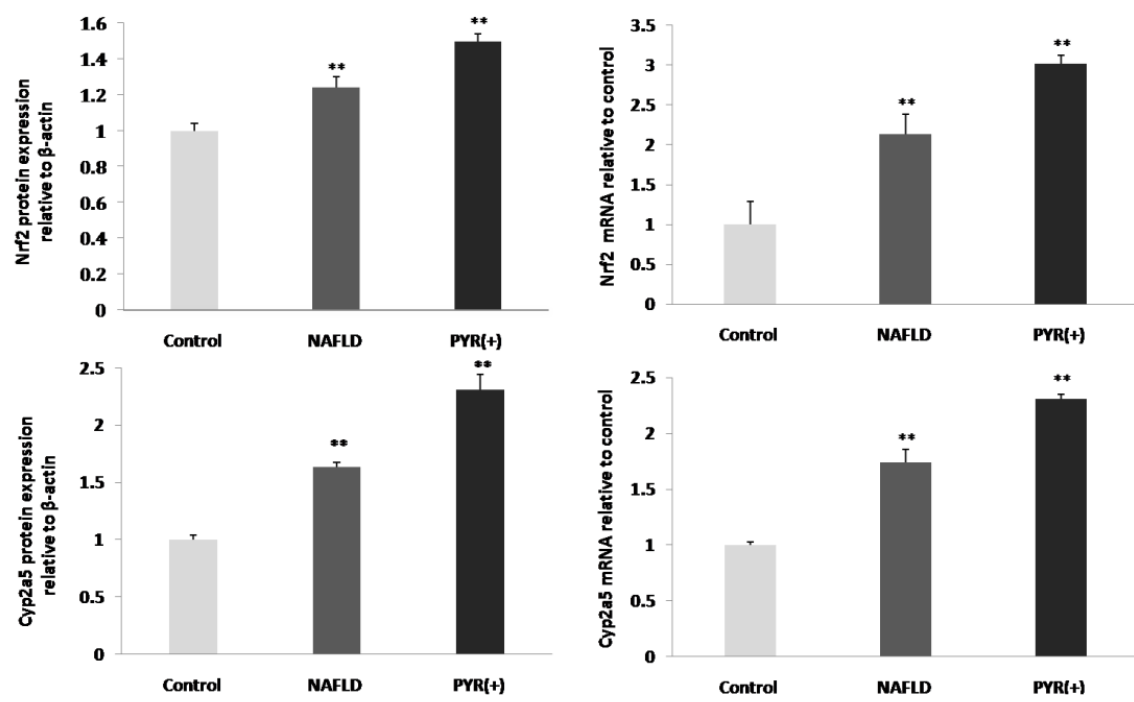

Figure 3: NAFLD and pyrazole increase Nrf2 and Cyp2a5 protein expression in vivo (Western blot (A) and densitometric analysis (B) of Cyp2a5 and Nrf2 protein expression in liver obtained from mice treated with control, high-fat diet and pyrazole. Values represent the mean $\pm S D(n=3)$ expressed as a percent relative intensity of control. Values represent the mean $\pm S D(n=3)$. Values are significantly different from controls $\left({ }^{* *} P<0.01\right)$. (C) $C O H$ activity was assessed in liver obtained from mice treated with control, high-fat diet and pyrazole. (D) Cyp2a5 and Nrf2 mRNA levels were quantified by RT-qPCR and normalized to GAPDH: average expression obtained from mice treated with control, high-fat diet and pyrazole. Values represent the mean $\pm S D(n=3)$. Values are significantly different from controls $\left({ }^{\star *} P<0.01\right)$ ). 


\section{A}

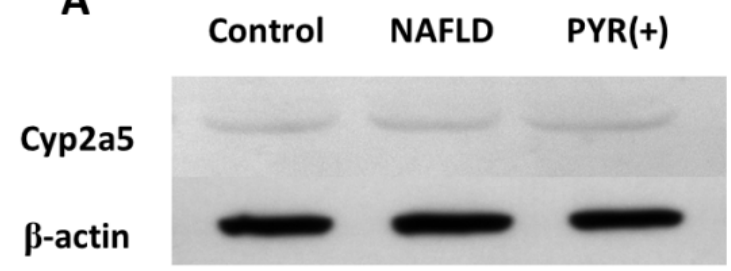

Nrf2(-)-

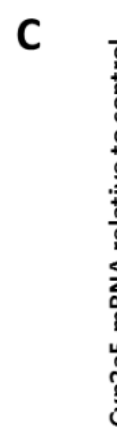

D

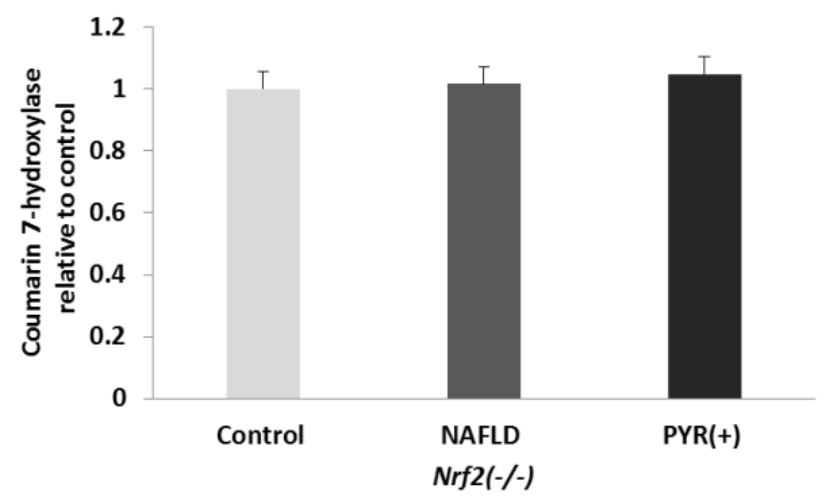

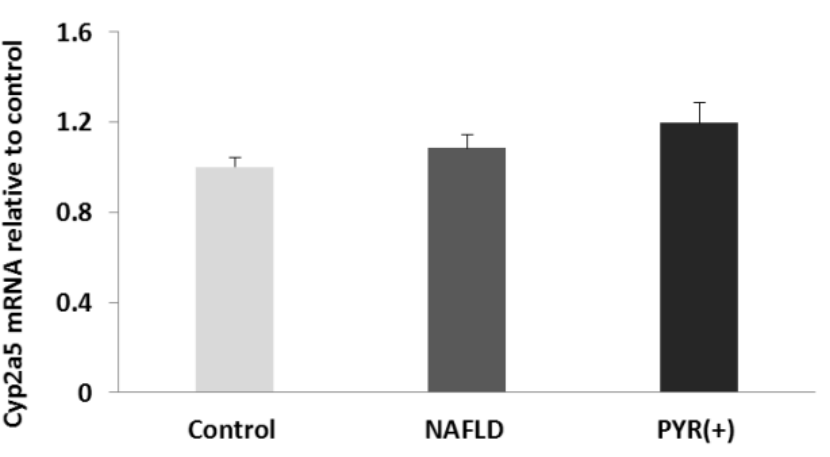

Nrf2(-/-)

Figure 4: Cyp2a5 protein expression, mRNA levels and coumarin 7-hydroxylase activity in the Nrf2(-/-) mice (Western blot (A) and densitometric analysis (B) of Cyp2a5 and Nrf2 protein expression in liver obtained from mice treated with Control, high-fat diet and pyrazole. (C) Cyp2a5 mRNA levels were quantified by RT-qPCR and normalized to GAPDH. (D) $\mathrm{COH}$ activitywas assessed in liver obtained from mice treated with control, high-fat diet and pyrazole. Values represent the mean \pm SD $(n=3)$ expressed as a percent relative intensity of control).

IP: Nrf2

\section{A}

IB: Cyp2a5

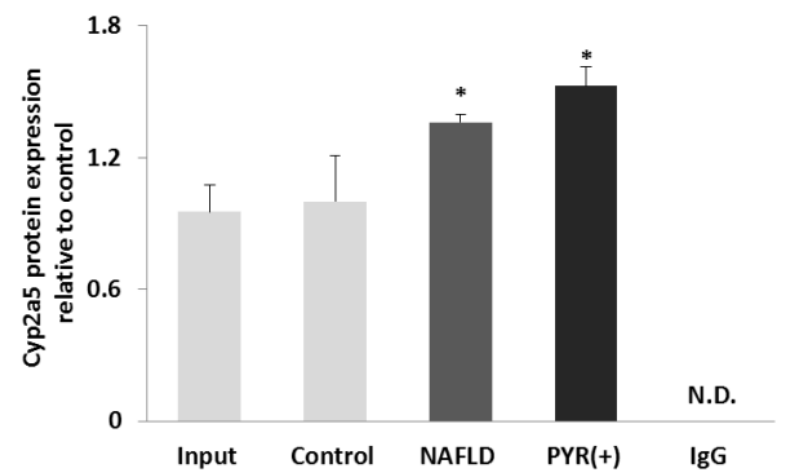

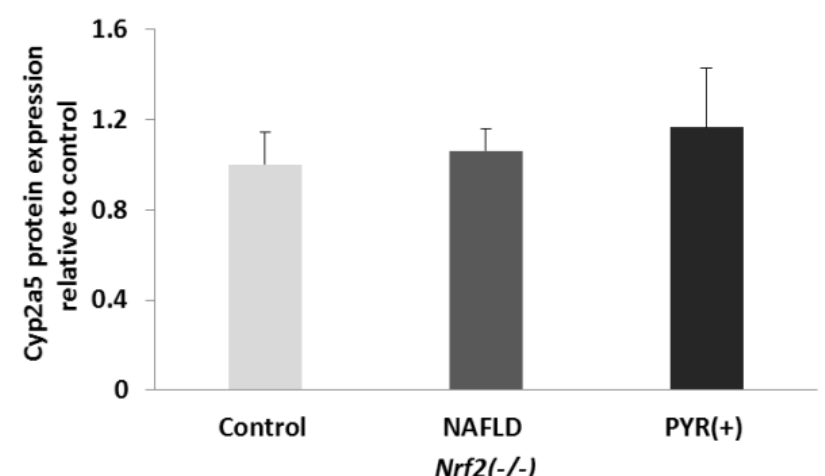

IP: Cyp2a5
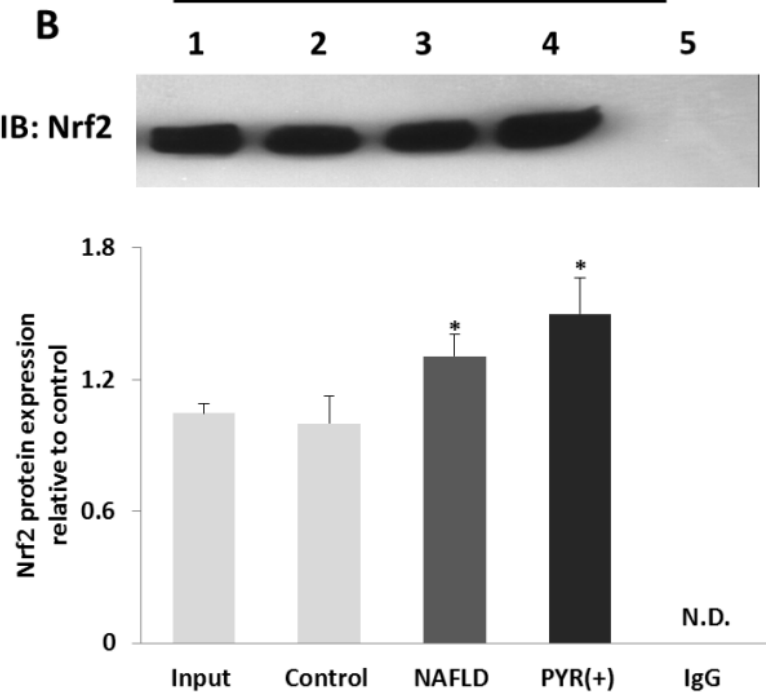

Figure 5: Nrf2 protein expresses along with Cyp2a5 protein interactively in WT mice and NAFLD induces the two protein expression ((A) Co-immunoprecipitated with anti-Nrf2 and Western blot with anti-Cpy2a5 showing expression of Cyp2a5. (B) Co-immunoprecipitated with anti-Cpy2a5 and Western blot with anti-Nrf2 showing expression of Nrf2. Relative amount of the co-immunoprecipitation complex Nrf2 and Cyp2a5 valuated by the ratio of band optical density. All values represent the mean $\pm S D(n=3)$ against the control levels. Mean differences are significant from control group at $P<0.05\left(^{*}\right)$. (1) liver extracts (input) were used as positive control; (2) immunoprecipitates from wild-type control group mice livers; (3) immunoprecipitates from high-fat diet group mice livers; (4) immunoprecipitates from pyrazole group mice livers; (5) immunoprecipit ates with IgG used as negative control; IP, co-immunoprecipitation; IB: immunoblot). 


\section{Discussion}

In this study, we found that feeding mice a high-fat diet results in an increase in Cyp2a5 levels and catalytic activity compared with those in controls. In addition, the observation of no change in the expression of Cyp2a5 mRNA in the liver of treated knock-out (Nrf2 $2^{-/)}$mice, in contrast to the changes seen in the wild-type $\left(\mathrm{Nrf2^{+/+ }}\right)$ mice suggests that NAFLD induction of Cyp2a5 requires the transcription factor Nrf2. This is consistent with the observations that pyrazole and heavy metals were ineffective in inducing Cyp2a 5 in $\mathrm{Nrf2}^{-/-}$mice. These results suggest that NAFLD-induced the Cyp2a5 expression in mice is due to the Nrf2.

Nrf2 regulates many important antioxidant genes and phase II detoxification genes. Oxidative stress plays an important role in NAFLD $[19,20]$. However, little is known about changes of Nrf2 in nonalcoholic fatty liver disease. The study shows that in chronic highfat-fed mice, liver Nrf2 protein and mRNA expression is increased. Induction of Nrf2 by injecting pyrazole into mice also increased Cyp2a5 protein expression. Nrf2 levels were elevated 1.5 fold in mice after pyrazole treatment. These increases were associated with similar increases in Cyp2a5 protein levels. These results demonstrate that Cyp2a5 is activated by increased expression of Nrf2 in pyrazole treated group. Levels of Nrf2 were also elevated in the livers of mice high-fat diet, and it is interesting to speculate that this increase may play a role in the elevated mRNA levels of Cyp2a5 caused by high-fat treatment.

Recently it was found that Nrf2 can regulate Cyp2a5 induction, which contains an ARE in its promoter [21]. Pyrazole-induced Cyp2a5 was lower in $\mathrm{Nrf2}^{-/-}$mice, compared with that in $\mathrm{Nrf2}^{+/+}$mice, suggesting that Cyp2a5 induction by pyrazole was mediated by Nrf2. Cyp2a5 induction by heavy metals including $\mathrm{CdCl} 2$ is also dependent on Nrf2 [22,23]. Ethanol induces Cyp2a5, at least in part, at the level of transcription because ethanol increased Cyp2a5 mRNA levels [24]. We found that the expression and activity of Cyp2a 5 were no difference in the $\mathrm{Nrf2}^{-}$mice (Figure 4). In addition to Nrf2, several other transcription factors have been implicated in the basal and inducible expression of the Cyp2a5 gene [25-27]. It is possible that one or some of those transcription factors may take part in regulating NAFLD induction of Cyp2a5, especially because Cyp2a5 was still induced by NAFLD in $\mathrm{Nrf2}^{--}$mice although the extent of induction was lower compared with that in $\mathrm{Nrf2} 2^{+/+}$mice.

We believe the most novel information in this study is the close association between levels of Nrf2 and Cyp2a5 and the fact that the induction of Cyp2a 5 by high-fat diet seems to be potentiated by the induction of Nrf2. Increased expression of Nrf2 has been reported in livers of mice fed a high-fat diet for 12 weeks [28]. In the current study, the Nrf2 signaling pathway was activated by the high-fat diet as indicated by increased nuclear accumulation of Nrf2 proteins (Figure 2). Immunohistochemical staining showed that the distribution of the elevated Nrf2 in the nucleus. As shown in Figure 1, we observed that $\mathrm{Nrf} 2$ was translocated from the cytoplasm into the nucleus in the highfat diet and pyrazole treated group. However, the control group neither decreased the Nrf2 levels in the cytoplasmic fraction or increased it as great as in the livers treated with high-fat diet or pyrazole observed in the nuclear fraction. Many of these can respond to the Nrf2 pathway and Nrf2 protein preferentially accumulated in the high-fat diet and pyrazole treated group as compared to control liver nuclei.

In summary, we have investigated the molecular mechanism of NAFLD elevates Cyp2a 5 protein expression and mRNA levels. We first validated a direct interaction of Nrf2 with Cyp2a5. Co-IP experiments showed that Nrf2 interacts directly with Cyp2a5 supplies a novel insight of a potential role of $\mathrm{Nrf} 2$ and suggests a possible mechanism of Nrf2 participation in the regulation of Cyp2a5 in NAFLD.

\section{Authors' Contributions}

YC and Pro. XZ designed the experiments and contributed to the description and writing. QW contributed to feed mice and did animal treatments, and contributed to the tissue collected. CW contributed to co-immunoprecipitation. GL contributed to the data analysis. $\mathrm{XY}$ and YQ contributed to the immunohistochemical staining. YC contributed to the RT-PCR assay. All authors read and approved the final manuscript.

\section{Acknowledgments}

We thank Dr H. Raunio (University of Kuopio, Kuopio, Finland) for generously providing antibodies against Cyp2a5.

\section{Funding}

This work was supported by The Natural Science Foundation of China, Grant No. 31172369 .

\section{References}

1. Bellentani S, Bedogni G, Miglioli L, Tiribelli C (2004) The epidemiology of fatty liver. European journal of gastroenterology \& hepatology 16:1087-1093.

2. Pessayre D, Berson A, Fromenty B, Mansouri A (2001) Mitochondria in steatohepatitis. Seminars in liver disease 21: 57-69.

3. Nelson DR, Zeldin DC, Hoffman SM, Maltais LJ, Wain HM, et al. (2004) Comparison of cytochrome P450 (CYP) genes from the mouse and human genomes, including nomenclature recommendations for genes, pseudogenes and alternative-splice variants. Pharmacogenetics 14:1-18.

4. Hahnemann B, Salonpaa P, Pasanen M, Maenpaa J, Honkakoski P, et al (1992) Effect of pyrazole, cobalt and phenobarbital on mouse liver cytochrome P-450 2a-4/5 (Cyp2a-4/5) expression. The Biochemical journal 286: 289-294.

5. Salonpaa P, Iscan M, Pasanen M, Arvela P, Pelkonen O, et al. (1992) Ceriuminduced strain-dependent increase in Cyp2a-4/5 (cytochrome P4502a-4/5) expression in the liver and kidneys of inbred mice. Biochemical pharmacology 44: $1269-1274$

6. Wastl UM, Rossmanith W, Lang MA, Camus-Randon AM, Grasl-Kraupp B, et al. (1998) Expression of cytochrome P450 2A5 in preneoplastic and neoplastic mouse liver lesions. Molecular carcinogenesis 22: 229-234

7. Raunio H, Juvonen R, Pasanen M, Pelkonen O, Paakko P, et al. (1998) Cytochrome P4502A6 (CYP2A6) expression in human hepatocellular carcinoma. Hepatology 27: 427-432.

8. Camus-Randon AM, Raffalli F, Bereziat JC, McGregor D, Konstandi M et al. (1996) Liver injury and expression of cytochromes P450: evidence that regulation of CYP2A5 is different from that of other major xenobiotic metabolizing CYP enzymes. Toxicology and applied pharmacology 138: 140148.

9. Gilmore WJ, Kirby GM (2004) Endoplasmic reticulum stress due to altered cellular redox status positively regulates murine hepatic CYP2A5 expression. The Journal of pharmacology and experimental therapeutics 308: 600-608.

10. Gong P, Cederbaum Al (2006) Nrf2 is increased by CYP2E1 in rodent liver and HepG2 cells and protects against oxidative stress caused by CYP2E1. Hepatology 43: 144-1453.

11. Abu-Bakar A, Satarug S, Marks GC, Lang MA, Moore MR (2004) Acute cadmium chloride administration induces hepatic and renal CYP2A5 mRNA protein and activity in the mouse: involvement of transcription factor NRF2. Toxicology letters 148: 199-210.

12. Jaiswal AK (2004) Nrf2 signaling in coordinated activation of antioxidant gene expression. Free radical biology \& medicine 36: 1199-1207.

13. Zhang DD (2006) Mechanistic studies of the Nrf2-Keap1 signaling pathway. Drug metabolism reviews 38: 769-89.

14. Nakata K, Tanaka Y, Nakano T, Adachi T, Tanaka H, et al. (2006) Nuclear receptor-mediated transcriptional regulation in Phase I, II, and III xenobiotic metabolizing systems. Drug metabolism and pharmacokinetics 21: 437-457. 
Citation: Cui Y, Wang Q, Wang C, Yi X, Qi Y, et al. (2015) Role of Nuclear Factor Erythroid 2-Like 2 in the Induction of Cytochrome P450 2a5 in Vivo of Nonalcoholic Fatty Liver Disease. J Diabetes Metab 6: 488. doi:10.4172/2155-6156.1000488

15. Wang X, Cao Y, Fu Y, Guo G, Zhang X (2011) Liver fatty acid composition in mice with or without nonalcoholic fatty liver disease. Lipids in health and disease 10: 234 .

16. Lu Y, Gong P, Cederbaum Al (2008) Pyrazole induced oxidative liver injury independent of CYP2E1/2A5 induction due to Nrf2 deficiency. Toxicology 252: 9-16.

17. Seglen PO (1976) Preparation of isolated rat liver cells. Methods in cell biology 13: $29-83$.

18. Aitio A (1978) A simple and sensitive assay of 7-ethoxycoumarin deethylation. Analytical biochemistry 85: 488-491.

19. Nordmann R, Ribiere C, Rouach H (1992) Implication of free radical mechanisms in ethanol-induced cellular injury. Free radical biology \& medicine 12: $219-240$.

20. Tsukamoto H, Lu SC (2001) Current concepts in the pathogenesis of alcoholic liver injury. FASEB journal: official publication of the Federation of American Societies for Experimental Biology 15: 1335-1349.

21. Lamsa V, Levonen AL, Leinonen H, Yla-Herttuala S, Yamamoto M, et al. (2010) Cytochrome P450 2A5 constitutive expression and induction by heavy metals is dependent on redox-sensitive transcription factor Nrf2 in liver. Chemical research in toxicology 23: 977-985.

22. Abu-Bakar A, Lamsa V, Arpiainen S, Moore MR, Lang MA, et al. (2007)
Regulation of CYP2A5 gene by the transcription factor nuclear factor (erythroid-derived 2)-like 2. Drug metabolism and disposition: the biological fate of chemicals 35: 787-794.

23. Abu-Bakar A, Moore MR, Lang MA (2005) Evidence for induced microsomal bilirubin degradation by cytochrome P450 2A5. Biochemical pharmacology 70 : 1527-1535.

24. Lu Y, Zhuge J, Wu D, Cederbaum Al (2011) Ethanol induction of CYP2A5 permissive role for CYP2E1. Drug metabolism and disposition: the biological fate of chemicals 39: 330-336.

25. Arpiainen S, Raffalli-Mathieu F, Lang MA, Pelkonen O, Hakkola J (2005) Regulation of the Cyp2a5 gene involves an aryl hydrocarbon receptordependent pathway. Molecular pharmacology 67: 1325-1333.

26. Arpiainen S, Lamsa V, Pelkonen O, Yim SH, Gonzalez FJ, Hakkola J (2007) Aryl hydrocarbon receptor nuclear translocator and upstream stimulatory factor regulate Cytochrome P450 2a5 transcription through a common E-box site. Journal of molecular biology 369: 640-652.

27. Cai Y, Konishi T, Han G, Campwala KH, French SW, et al. (2002) The role of hepatocyte RXR alpha in xenobiotic-sensing nuclear receptor-mediated pathways. European journal of pharmaceutical sciences : official journal of the European Federation for Pharmaceutical Sciences 15: 89-96.

28. Kim S, Sohn I, Ahn JI, Lee KH, Lee YS (2004) Hepatic gene expression profiles in a long-term high-fat diet-induced obesity mouse model. Gene 340: 99-109. 